International Journal of Pure and Applied Mathematics

Volume 91 No. 4 2014, 515-531

ISSN: 1311-8080 (printed version); ISSN: 1314-3395 (on-line version)

url: http://www.ijpam.eu

doi: http://dx.doi.org/10.12732/ijpam.v91i4.8

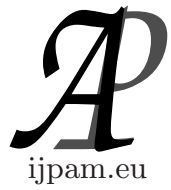

\title{
HOPF BIFURCATION OF AN EPIDEMIC MODEL WITH \\ A NONLINEAR BIRTH IN POPULATION \\ AND STAGE STRUCTURE
}

\author{
Yinying Zhang ${ }^{1}$, Jianwen $\mathrm{Jia}^{2} \S$ \\ School of Mathematics and Computer Science \\ Shanxi Normal University \\ Shanxi, Linfen, 041004, P.R. CHINA
}

\begin{abstract}
In this paper, an epidemic model involving a nonlinear birth in population and stage structure was studied. The stability of disease-free equilibrium was verified by Routh-Hurwitz criterion and LaSalle's invariance principle. We researched the existence of Hopf bifurcation and obtained the stability and direction of the Hopf bifurcation by using the normal theory and the center manifold theorem. In the end, numerical simulations were carried out to illustrate the main theoretical results and a brief discussion was given to conclude this work.
\end{abstract}

AMS Subject Classification: 34C25, 34K45, 92D25

Key Words: stage structure, Hopf bifurcation, center manifold theorem

\section{Introduction}

Mathematical modeling is of considerable importance in the study of epidemiology because it may provide understanding of the underlying mechanisms which influence the spread of disease and may suggest control strategies. Since Kermack and Mckendrick constructed a system of ODE [8] to study epidemiology in 1927, the concept of "compartment modeling" is used until now. Most of

Received: December 14, 2013

(C) 2014 Academic Publications, Ltd.

${ }^{\S}$ Correspondence author url: www.acadpubl.eu 
the research literature assumes that once infected, each susceptible individual (in the class S) becomes infectious (in the class I), instantaneously and later recovers (in the class $\mathrm{R}$ ) with a permanent or temporary acquired immunity. We usually call these compartmental models SIR models or SIRS models with each letter denoting a compartment to which an individual are belonged.

Stage-structure is a natural phenomenon and represents, for example, the division of a population into immature andmature individuals, and have received much attention in recent years [1, 10, 12, 14]. For some diseases, such as sexual diseases or bacterial infections, it is reasonable to consider the disease transmission in the adult population and neglect transmission in juveniles, while other infectious diseases such as measles, chickenpox can spread among children. If we use "compartment modeling" to study these infectious diseases, the stage structure is required to consider corresponding epidemic models. For example, Zhang, Liu and Teng [14] proposed a delayed SIRS epidemic model with stage structure that a disease spreads among mature individuals and time delay. They investigated the stability of an endemic equilibrium and the direction of the Hopf bifurcation and stability of bifurcating periodic solutions.

\section{The Model}

Classical epidemic models assume that the total population size is constant, and concentrate on describing the spread of disease through the population. More recent models consider a variable population size, thus taking into account a longer time scale with disease causing death and reduced reproduction. For example, in paper [14] considers a nonlinear birth term $B(N)$, and finds that the form $B(N) N$ is important in determining the qualitative dynamics. In the absence of disease, the paper [2] assumes that the total population size $N$ changes according to a population growth equation

$$
\frac{\mathrm{d} N(t)}{\mathrm{d} t}=B(N) N-d N
$$

Here $d>0$ is the death rate constant, and $B(N) N$ is a birth rate function with $B(N)$ satisfying with following basic assumptions for $N \in(0, \infty)$ :

(A1) $B(N)>0$;

(A2) $B(N)$ is continuously differentiable with $B^{\prime}(N)<0$;

(A3) $B\left(0^{+}\right)>d>B(\infty)$. 
Based on the above description, we choose a $B(N)=\frac{C}{N}+B$. It is clear that $B(N)$ meets (A1) and (A2). Because of (A3), we can choose $B$, $d$ satisfying $B<d$. In [14], they suppose that only susceptible individual have the ability to give birth. But for reality, all adults have the ability to give birth. Under these assumptions, we consider the following epidemic model with nonlinear birth in population and nonlinear incidence.

$$
\left\{\begin{array}{l}
\frac{\mathrm{d} J(t)}{\mathrm{d} t}=\left(\frac{C}{A(t)}+B\right) A(t)-(\omega+d) J(t) \\
\frac{\mathrm{d} S(t)}{\mathrm{d} t}=\omega J(t)-d S(t)-\frac{\beta S(t) I(t-\bar{\tau})}{1+\alpha I(t-\bar{\tau})}+\delta R(t) \\
\frac{\mathrm{d} I(t)}{\mathrm{d} t}=\frac{\beta S(t) I(t-\bar{\tau})}{1+\alpha I(t-\bar{\tau})}-(d+\gamma+\mu) I(t) \\
\frac{\mathrm{d} R(t)}{\mathrm{d} t}=\gamma I(t)-(d+\delta) R(t)
\end{array}\right.
$$

where $A(t)=S(t)+I(t)+R(t), N(t)=J(t)+A(t)=J(t)+S(t)+I(t)+R(t)$ and $C, B, \omega, d, \beta, \alpha, \delta, \gamma, \mu$ are positive. $J(t), S(t), I(t), R(t)$ denote densities of juveniles, susceptible, infected and recover population stage at time $t$, respectively. $A(t)$ represent the density of adults at time $t$ and $N(t)$ is the density of total population. $C$ is immigrants, $B$ is the birth rate and $d$ is the natural death rate. $\omega$ is the conversion rate from juvenile to mature individual, that is $\frac{1}{\omega}$ is the average period to maturity of juvenile. $\beta$ is contact rate between the susceptible and the infection. $\mu$ is the death rate due to the disease. $\gamma$ is the recovery rate. $\delta$ is the rate at which the recovered individuals return to the susceptible class. $\tau$ is the time delay.

The initial conditions for system (2.1) are

$$
\begin{aligned}
& \left(\phi(\theta), \psi_{1}(\theta), \psi_{2}(\theta), \psi_{2}(\theta)\right) \in C_{+}=C\left([-\tau, 0], \mathbb{R}_{+}^{4}\right), \\
& \phi(0)>0, \quad \psi_{i}(0)>0, \quad i=1,2,3
\end{aligned}
$$

where $\mathbb{R}_{+}^{4}=\left\{\left(x_{1}, x_{2}, x_{3}, x_{4}\right) \in \mathbb{R}^{4}: x_{i} \geq 0, i=1,2,3,4\right\}$.

Theorem 1. For any solution $J(t), S(t), I(t), R(t)$ of system (2.1) with initial conditions (2.2), $J(t)<M, S(t)<M, I(t)<M, R(t)<M$ for all large $t$, where $M=\frac{C}{d-B}$.

Proof.We omit the simple proof.

For the sake of simplicity, we put in dimensionless form the system (2.1) by rescaling the variables

$$
j=\frac{\omega+d}{C} J, \quad s=\frac{B}{C} S, \quad i=\frac{B}{C} I, \quad r=\frac{B}{C} R
$$

and use the dimensionless time scale $\bar{t}=(\omega+d)$ t. However, to avoid heavy 
notation, $\bar{t}$ will be substituted by $t$. Then the system (2.1) is transformed into

$$
\left\{\begin{array}{l}
\frac{\mathrm{d} j(t)}{\mathrm{d} t}=1+s+i+r-j \\
\frac{\mathrm{d} s(t)}{\mathrm{d} t}=a j(t)-b_{1} s(t)-\frac{\beta_{1} s(t) i(t-\tau)}{1+\alpha_{1} i(t-\tau)}+\delta_{1} r(t) \\
\frac{\mathrm{d} i(t)}{\mathrm{d} t}=\frac{\beta_{1} s(t) i(t-\tau)}{1+\alpha_{1} i(t-\tau)}-b_{2} i(t) \\
\frac{\mathrm{d} r(t)}{\mathrm{d} t}=\gamma_{1} i(t)-b_{3} r(t)
\end{array}\right.
$$

where $\quad a=\frac{\omega B}{(\omega+d)^{2}}, \quad b_{1}=\frac{d}{\omega+d}, \quad \beta_{1}=\frac{\beta C}{(\omega+d) B}, \quad \alpha_{1}=\frac{\alpha C}{B}, \quad b_{2}=$ $\frac{d+\gamma+\mu}{\omega+d}, \quad \delta_{1}=\frac{\delta}{\omega+d}$,

$$
\gamma_{1}=\frac{\gamma}{\omega+d}, \quad b_{3}=\frac{d+\delta}{\omega+d}=b_{1}+\delta_{1}, \quad \tau=(\omega+d) \bar{\tau}
$$

This paper is organized as following: In the next section, we obtain the basic reproduction number by the next generation method and the existence of equilibriums. We verify the stability of disease-free equilibrium by RouthHurwitz criterion and LaSalle's invariance principle. Then we focus on the local stability of the endemic equilibrium and the existence of the Hopf bifurcation. In Section 4, we obtain the stability and direction of the Hopf bifurcation by using the normal theory and the center manifold theorem. Numerical simulations are carried out in Section 5 to illustrate the main theoretical results. A brief discussion is given in last part to conclude this work.

\section{The Existence and Stability of Equilibria}

\subsection{The Existence of Equilibria and the Stability of the Disease-Free Equilibrium}

It is easy to obtain the disease-free equilibrium $E_{0}=\left(j_{0}, s_{0}, 0,0\right)=\left(\frac{b_{1}}{b_{1}-a}, \frac{a}{b_{1}-a}\right.$, $0,0)$. Then, we define the basic reproduction number $\mathscr{R}_{0}$ of our model by directly using the next generation method presented in Diekmann et al. [5] and P.van den Driessche and James Watmough [6].

Then

$$
\mathscr{R}_{0}=\frac{a \beta_{1}}{\left(b_{1}-a\right) b_{2}} .
$$

Theorem 2. When $\mathscr{R}_{0}<1$, there exists the unique disease-free equilibrium $E_{0}$. When $\mathscr{R}_{0}>1$, there also exist a endemic equilibrium $E^{*}=$ 
$\left(j^{*}, s^{*}, i^{*}, r^{*}\right)$, where $j^{*}=1+\frac{b_{2}\left(1+\alpha_{1} i^{*}\right)}{\beta_{1}}+i^{*}+\frac{\gamma_{1}}{b_{3}} i^{*}$, $s^{*}=\frac{b_{2}\left(1+\alpha_{1} i^{*}\right)}{\beta_{1}}, \quad r^{*}=\frac{\gamma_{1}}{b_{3}} i^{*}$ and $\quad i^{*}=\frac{\left[a\left(\beta_{1}+b_{2}\right)-b_{1} b_{2}\right] b_{3}}{\alpha_{1} b_{2} b_{3}\left(b_{1}-a\right)+\beta_{1}\left(b_{2} b_{3}-a b_{3}-a \gamma_{1}-\delta_{1} \gamma_{1}\right)}$.

Proof.We omit the simple proof.

Then we find the characteristic equation of any equilibrium $\bar{E}=(\bar{j}, \bar{s}, \bar{i}, \bar{r})$ as follows:

$$
\operatorname{det}(\lambda I-J(\bar{E})) \text {. }
$$

Theorem 3. When $\mathscr{R}_{0}<1$, the disease-free equilibrium $E_{0}$ is locally asymptotically stable for all $\tau \geq 0$; When $\mathscr{R}_{0}>1, E_{0}$ is unstable for all $\tau \geq 0$.

Proof.We omit the simple proof.

\subsection{The Locally Stability of the Endemic Equilibrium}

From the above results, when $\mathscr{R}_{0}>1, E_{0}$ is unstable for all $\tau \geq 0$, and at the same time, an endemic equilibrium $E^{*}$ emerges. Now we focus on the stability of $E^{*}$.

The characteristic equation of system (2.3) at $E^{*}$ is of the form

$$
\lambda^{4}+A_{1} \lambda^{3}+A_{2} \lambda^{2}+A_{3} \lambda+A_{4}-\left[\lambda^{3}+B_{1} \lambda^{2}+B_{2} \lambda+B_{3}\right] B_{4} e^{(-\lambda \tau)}=0 .
$$

where

$$
\begin{gathered}
A_{1}=1+b_{1}+b_{2}+b_{3}+\frac{\beta_{1} i^{*}}{1+\alpha_{1} i^{*}} \\
A_{2}=b_{1}+b_{2}+b_{3}+b_{1} b_{2}+b_{1} b_{3}+b_{2} b_{3}-a+\frac{\left(b_{2}+b_{3}+1\right) \beta_{1} i^{*}}{1+\alpha_{1} i^{*}}, \\
A_{3}=b_{1} b_{2}+b_{1} b_{3}+b_{2} b_{3}+b_{1} b_{2} b_{3}-a\left(b_{2}+b_{3}\right)+\frac{\left(b_{2}+b_{3}-a+b_{2} b_{3}-\delta_{1} \gamma_{1}\right) \beta_{1} i^{*}}{1+\alpha_{1} i^{*}} \\
A_{4}=b_{1} b_{2} b_{3}-a b_{2} b_{3}+\frac{\left[b_{3}\left(b_{2}-a\right)-\left(a+\delta_{1}\right) \gamma_{1}\right] \beta_{1} i^{*}}{1+\alpha_{1} i^{*}} \\
B_{1}=1+b_{1}+b_{3}, \quad B_{2}=b_{1}+b_{3}-a+b_{1} b_{3} \\
B_{3}=b_{3}\left(b_{1}-a\right), \quad B_{4}=\frac{\beta_{1} s^{*}}{\left(1+\alpha_{1} i^{*}\right)^{2}}
\end{gathered}
$$


Theorem 4 . When $\mathscr{R}_{0}>1, E^{*}$ is locally asymptotically stable for $\tau=0$.

Proof. When $\tau=0$, the characteristic equation at $E^{*}$ is of the form:

$$
\lambda^{4}+m_{1} \lambda^{3}+m_{2} \lambda^{2}+m_{3} \lambda+m_{4}=0 .
$$

where

$$
\begin{aligned}
m_{1} & =A_{1}-B_{4}=1+b_{1}+b_{2}+b_{3}+\frac{\beta_{1} i^{*}}{1+\alpha_{1} i^{*}}-\frac{\beta_{1} s^{*}}{\left(1+\alpha_{1} i^{*}\right)^{2}} ; \\
m_{2} & =A_{2}-B_{1} B_{4}=b_{1}+b_{2}+b_{3}+b_{1} b_{2}+b_{1} b_{3}+b_{2} b_{3}-a \\
& +\frac{\left(b_{2}+b_{3}+1\right) \beta_{1} i^{*}}{1+\alpha_{1} i^{*}}-\frac{\left(1+b_{1}+b_{3}\right) \beta_{1} s^{*}}{\left(1+\alpha_{1} i^{*}\right)^{2}} \\
m_{3} & =A_{3}-B_{2} B_{4} \\
& =b_{1} b_{2}+b_{1} b_{3}+b_{2} b_{3}+b_{1} b_{2} b_{3}-a\left(b_{2}+b_{3}\right) \\
& +\frac{\left(b_{2}+b_{3}-a+b_{2} b_{3}-\delta_{1} \gamma_{1}\right) \beta_{1} i^{*}}{1+\alpha_{1} i^{*}}-\frac{\left(b_{1}+b_{3}-a+b_{1} b_{3}\right) \beta_{1} s^{*}}{\left(1+\alpha_{1} i^{*}\right)^{2}} \\
m_{4} & =A_{4}-B_{3} B_{4}=b_{1} b_{2} b_{3}-a b_{2} b_{3}+\frac{\left[b_{3}\left(b_{2}-a\right)-\left(a+\delta_{1}\right) \gamma_{1}\right] \beta_{1} i^{*}}{1+\alpha_{1} i^{*}}-\frac{b_{3}\left(b_{1}-a\right) \beta_{1} s^{*}}{\left(1+\alpha_{1} i^{*}\right)^{2}}
\end{aligned}
$$

For the fact that $\frac{\beta_{1} s^{*}}{\left(1+\alpha_{1} i^{*}\right)^{2}}=\frac{b_{2}}{1+\alpha_{1} i^{*}}<b_{2}$, we can obtain $m_{k}>0(k=1,2,3,4)$. Then

$$
\begin{aligned}
\triangle_{1}= & m_{1}, \quad \triangle_{3}=m_{3}\left(m_{1} m_{2}-m_{3}\right)-m_{1}^{2} m_{4} . \\
\triangle_{2}= & m_{1} m_{2}-m_{3} \\
= & {\left[1+b_{1}+b_{2}+b_{3}+\frac{\beta_{1} i^{*}}{1+\alpha_{1} i^{*}}-\frac{\beta_{1} s^{*}}{\left(1+\alpha_{1} i^{*}\right)^{2}}\right] } \\
& {\left[b_{1}+b_{2}+b_{3}+b_{1} b_{2}+b_{1} b_{3}+b_{2} b_{3}-a+\frac{\left(b_{2}+b_{3}+1\right) \beta_{1} i^{*}}{1+\alpha_{1} i^{*}}-\frac{\left(1+b_{1}+b_{3}\right) \beta_{1} s^{*}}{\left(1+\alpha_{1} i^{*}\right)^{2}}\right] } \\
& -\left[b_{1} b_{2}+b_{1} b_{3}+b_{2} b_{3}+b_{1} b_{2} b_{3}-a\left(b_{2}+b_{3}\right)\right. \\
& \left.+\frac{\left(b_{2}+b_{3}-a+b_{2} b_{3}-\delta_{1} \gamma_{1}\right) \beta_{1} i^{*}}{1+\alpha_{1} i^{*}}-\frac{\left(b_{1}+b_{3}-a+b_{1} b_{3}\right) \beta_{1} s^{*}}{\left(1+\alpha_{1} i^{*}\right)^{2}}\right]
\end{aligned}
$$

We obtain $\triangle_{k}>0(k=1,2,3)$ by directly comparing. Using Routh-Hurwitz criterion, we can obtain that when $\mathscr{R}_{0}>1$, the endemic equilibrium $E^{*}$ is locally asymptotically stable for all $\tau=0$.

\subsection{Hopf Bifurcation}

Now we discuss that when $\tau>0$, a Hopf bifurcation may emerge if the parameters satisfy some conditions. Assume that for some $\tau>0, i \xi(\xi>0)$ is a root of (3.2), which implies

$$
\xi^{4}-i A_{1} \xi^{3}-A_{2} \xi^{2}+i A_{3} \xi+A_{4}-\left[-i \xi^{3}-B_{1} \xi^{2}+i B_{2} \xi+B_{3}\right] B_{4} e^{(-i \xi \tau)}=0 .
$$


Separating real and imaginary parts, we have

$$
\begin{aligned}
& \xi^{4}-A_{2} \xi^{2}+A_{4}=B_{4}\left(B_{2} \xi-\xi^{3}\right) \sin (\xi \tau)+B_{4}\left(B_{3}-B_{1} \xi^{2}\right) \cos (\xi \tau) \\
& -A_{1} \xi^{3}+A_{3} \xi=B_{4}\left(B_{2} \xi-\xi^{3}\right) \cos (\xi \tau)-B_{4}\left(B_{3}-B_{1} \xi^{2}\right) \sin (\xi \tau) .
\end{aligned}
$$

It follows from (3.4) that

$$
\xi^{8}+p \xi^{6}+q \xi^{4}+r \xi^{2}+s=0 .
$$

where

$$
\begin{array}{lc}
p=A_{1}^{2}-2 A_{2}-B_{4}^{2} ; & q=A_{2}^{2}+2 A_{4}-2 A_{1} A_{3} \\
r=A_{3}^{2}-2 A_{2} A_{4}+2 B_{1} B_{3} B_{4}^{2}-B_{2}^{2} B_{4}^{2} ; & s=B_{4}^{2} B_{4}^{2}+2 B_{3}^{2} B_{4}^{2} .
\end{array}
$$

Letting $z=\xi^{2}$, the equation (3.5) becomes

$$
z^{4}+p z^{3}+q z^{2}+r z+s=0
$$

Denote $h(z)=z^{4}+p z^{3}+q z^{2}+r z+s$. Then we have

$$
h^{\prime}(z)=4 z^{3}+3 p z^{2}+2 q z+r .
$$

Theorem 5. Suppose that (3.7) has no positive roots, then when $\mathscr{R}_{0}>1$, $E^{*}$ is locally asymptotically stable for all $\tau \geq 0$.

Suppose that (3.7) has positive roots. Without loss of generality, we assume that it has $\widetilde{k}(1 \leq \widetilde{k} \leq 4)$ positive real roots, denote by $z_{1}<z_{2}<\ldots<z_{\widetilde{k}}$, respectively. Then (3.5) has $\widetilde{k}$ positive real roots

$$
\xi_{1}=\sqrt{z_{1}}, \xi_{2}=\sqrt{z_{2}}, \ldots, \xi_{\widetilde{k}}=\sqrt{z_{\widetilde{k}}} .
$$

From (3.4), we have

$$
\cos (\xi \tau)=\frac{\left(B_{3}-B_{1} \xi^{2}\right)\left(\xi^{4}-A_{2} \xi^{2}+A_{4}\right)+\left(B_{2} \xi-\xi^{3}\right)\left(-A_{1} \xi^{3}+A_{3} \xi\right)}{B_{4}\left(B_{3}-B_{1} \xi^{2}\right)^{2}+B_{4}\left(B_{2} \xi-\xi^{3}\right)^{2}} .
$$

Thus, if we denote

$$
\begin{aligned}
& \tau_{k}^{(j)}=\frac{1}{\xi_{k}}\left\{\cos ^{-1}\left[\frac{\left(B_{3}-B_{1} \xi_{k}^{2}\right)\left(\xi_{k}^{4}-A_{2} \xi_{k}^{2}+A_{4}\right)+\left(B_{2} \xi_{k}-\xi_{k}^{3}\right)\left(-A_{1} \xi_{k}^{3}+A_{3} \xi_{k}\right)}{B_{4}\left(B_{3}-B_{1} \xi_{k}^{2}\right)^{2}+B_{4}\left(B_{2} \xi_{k}-\xi_{k}^{3}\right)^{2}}\right]\right. \\
& +2 j \pi\} \text {, }
\end{aligned}
$$


where $k=1,2, \ldots, \widetilde{k} ; j=0,1,2, \ldots$, then $\pm i \xi_{k}$ are a pair of purely imaginary roots of the equation $(3.2)$ with $\tau_{k}^{(j)}$.

Let $m(\tau)=\zeta(\tau)+i \xi(\tau)$ be the root of (4.1) near $\tau=\tau_{k}^{(j)}$ satisfying $\zeta\left(\tau_{k}^{(j)}\right)=$ $0, \xi\left(\tau_{k}^{(j)}\right)=\xi_{k}$. On substituting $m(\tau)$ into the equation (3.2) and calculating the derivative with respective to $\tau$, we obtain

$$
\begin{aligned}
& {\left[4 m^{3}(\tau)+3 A_{1} m^{2}(\tau)+2 A_{2} m(\tau)+A_{3}\right] \frac{\mathrm{d} m(\tau)}{\mathrm{d} \tau}-\left[3 m^{2}(\tau)+2 B_{1} m(\tau)+B_{2}\right.} \\
& \left.-\tau\left(m^{3}(\tau)+B_{1} m^{2}(\tau)+B_{2} m(\tau)+B_{3}\right)\right] B_{4} e^{(-m(\tau) \tau)} \frac{\mathrm{d} m(\tau)}{\mathrm{d} \tau} \\
= & -m(\tau)\left[m^{3}(\tau)+B_{1} m^{2}(\tau)+B_{2} m(\tau)+B_{3}\right] B_{4} e^{(-m(\tau) \tau)}
\end{aligned}
$$

Then, we can get

$$
\begin{aligned}
\left(\frac{\mathrm{d} m(\tau)}{\mathrm{d} \tau}\right)^{-1}= & \frac{3 m^{4}(\tau)+2 A_{1} m^{3}(\tau)+A_{2} m^{2}(\tau)-A_{4}}{\left.-m(\tau)^{2}\left[m^{3}(\tau)+B_{1} m^{2}(\tau)+B_{2} m(\tau)+B_{3}\right] B_{4} e^{(}-m(\tau) \tau\right)} \\
& +\frac{2 m^{3}(\tau)+B_{1} m^{2}(\tau)-B_{3}}{m(\tau)^{2}\left[m^{3}(\tau)+B_{1} m^{2}(\tau)+B_{2} m(\tau)+B_{3}\right]}-\frac{\tau}{m} \\
= & \frac{3 m^{4}+2 A_{1} m^{3}+A_{2} m^{2}-A_{4}}{-m^{2}\left[m^{4}+A_{1} m^{3}+A_{2} m^{2}+A_{3} m+A_{4}\right]}+\frac{2 m^{3}+B_{1} m^{2}-B_{3}}{m^{2}\left(m^{3}+B_{1} m^{2}+B_{2} m+B_{3}\right)}-\frac{\tau}{m} .
\end{aligned}
$$

So

$$
\begin{aligned}
{\left[\frac{\mathrm{dRe} m(\tau)}{\mathrm{d} \tau}\right]_{\tau=\tau_{k}^{(j)}}^{-1}=} & \operatorname{Re}\left[\frac{3 m^{4}+2 A_{1} m^{3}+A_{2} m^{2}-A_{4}}{-m^{2}\left[m^{4}+A_{1} m^{3}+A_{2} m^{2}+A_{3} m+A_{4}\right.}\right]_{\tau=\tau_{k}^{(j)}} \\
& +\operatorname{Re}\left[\frac{2 m^{3}+B_{1} m^{2}-B_{3}}{m^{2}\left(m^{3}+B_{1} m^{2}+B_{2} m+B_{3}\right)}\right]_{\tau=\tau_{k}^{(j)}} \\
= & \frac{\left(3 \xi_{k}^{4}-A_{2} \xi_{k}^{2}-A_{4}\right)\left(\xi_{k}^{4}-A_{2} \xi_{k}^{2}+A_{4}\right)+2 A_{1} \xi_{k}^{3}\left(A_{1} \xi_{k}^{3}-A_{3} \xi_{k}\right)}{\xi_{k}^{2}\left(\xi_{k}^{4}-A_{2} \xi_{k}^{2}+A_{4}\right)^{2}+\xi_{k}^{2}\left(A_{1} \xi_{k}^{3}-A_{3} \xi_{k}\right)^{2}} \\
& +\frac{\left(B_{3}+B_{1} \xi_{k}^{2}\right)\left(B_{3}-B_{1} \xi_{k}^{2}\right)-2 \xi_{k}^{3}\left(\xi_{k}^{3}-B_{2} \xi_{k}\right)}{\xi_{k}^{2}\left(B_{3}-B_{1} \xi_{k}^{2}\right)^{2}+\xi_{k}^{2}\left(\xi_{k}^{3}-B_{2} \xi_{k}\right)^{2}} \\
= & \frac{\left(3 z_{k}^{2}-A_{2} z_{k}-A_{4}\right)\left(z_{k}^{2}-A_{2} z_{k}+A_{4}\right)+2 A_{1} z_{k}^{2}\left(A_{1} z_{k}-A_{3}\right)}{z_{k}\left(z_{k}^{2}-A_{2} z_{k}+A_{4}\right)^{2}+z_{k}^{2}\left(A_{1} z_{k}-A_{3}\right)^{2}} \\
& +\frac{\left(B_{3}+B_{1} z_{k}\right)\left(B_{3}-B_{1} z_{k}\right)-2 z_{k}^{2}\left(z_{k}-B_{2}\right)}{z_{k}\left(B_{3}-B_{1} z_{k}\right)^{2}+z_{k}^{2}\left(z_{k}-B_{2}\right)^{2}}
\end{aligned}
$$

where, $z_{k}=\xi_{k}^{2}$.

It follows from (3.6), (3.7) that

$$
\begin{aligned}
& \left(z_{k}^{2}-A_{2} z_{k}+A_{4}\right)^{2}+z_{k}\left(A_{1} z_{k}-A_{3}\right)^{2} \\
= & z_{k}^{4}+\left(A_{1}^{2}-2 A_{2}\right) z_{k}^{3}+\left(A_{2}^{2}+2 A_{4}-2 A_{1} A_{3}\right) z_{k}^{2}+\left(A_{3}^{2}-2 A_{2} A_{4}\right) z_{k}+A_{4}^{2} \\
= & B_{4}^{2}\left(B_{1} z_{k}-B_{3}\right)^{2}+B_{4}^{2} z_{k}\left(z_{k}-B_{2}\right)^{2} .
\end{aligned}
$$

Then

$$
\left[\frac{\mathrm{dRe} m(\tau)}{\mathrm{d} \tau}\right]_{\tau=\tau_{k}^{(j)}}^{-1}=\frac{3 z_{k}^{4}+2 p z_{k}^{3}+q z_{k}^{2}-s}{z_{k}\left[B_{4}^{2}\left(B_{1} z_{k}-B_{3}\right)^{2}+B_{4}^{2} z_{k}\left(z_{k}-B_{2}\right)^{2}\right]}=\frac{h^{\prime}\left(z_{k}\right)}{\Gamma} .
$$


Where $\Gamma=B_{4}^{2}\left(B_{1} z_{k}-B_{3}\right)^{2}+B_{4}^{2} z_{k}\left(z_{k}-B_{2}\right)^{2}$. Because of $B_{3}^{2} B_{4}^{2}-A_{4}^{2}=-s=$ $z^{4}+p z^{3}+q z^{2}+r z$. So, $\operatorname{sign}\left[\frac{\mathrm{d} \zeta(\tau)}{\mathrm{d} \tau}\right]_{\tau=\tau_{k}^{(j)}}^{-1}=\operatorname{sign}\left[\frac{\mathrm{dRe} m(\tau)}{\mathrm{d} \tau}\right]_{\tau=\tau_{k}^{(j)}}^{-1}=\operatorname{sign}\left[\frac{h^{\prime}\left(z_{k}\right)}{\Gamma}\right]=$ $\operatorname{sign}\left[h^{\prime}\left(z_{k}\right)\right]$.

Thus, we obtain the following theorem.

Theorem 6. Suppose $z_{k}=\xi_{k}^{2}$ and $h^{\prime}\left(z_{k}\right) \neq 0$, then $\frac{\mathrm{d} \zeta\left(\tau_{k}^{(j)}\right)}{\mathrm{d} \tau} \neq 0$, and $\frac{\mathrm{d} \zeta\left(\tau_{k}^{(j)}\right)}{\mathrm{d} \tau}$ has same sign with $h^{\prime}\left(z_{k}\right)$.

Theorem 7. Assume that Eq.(3.7) has at least one simple positive root and $z^{*}$ is the last such root. Then there is a Hopf bifurcation for system (2.1) as $\tau$ passes upwards through $\tau^{*}$ leading to a periodic solution that bifurcates from $E^{*}$, where

$$
\begin{gathered}
\tau^{*}=\frac{1}{\sqrt{z^{*}}}\left\{\cos ^{-1}\left[\frac{\left(B_{3}-B_{1} z^{*}\right)\left(\left(z^{*}\right)^{2}-A_{2} z^{*}+A_{4}\right)+z^{*}\left(B_{2}-z^{*}\right)\left(-A_{1} z^{*}+A_{3}\right)}{B_{4}\left(B_{3}-B_{1} z^{*}\right)^{2}+B_{4} z^{*}\left(B_{2}-z^{*}\right)^{2}}\right]\right. \\
+2 j \pi\} .
\end{gathered}
$$

Remark. If there are four positive roots of (3.7), $z_{1}<z_{2}<z_{3}<z_{4}$, respectively, then $h^{\prime}\left(z_{1}\right)<0, h^{\prime}\left(z_{2}\right)>0, h^{\prime}\left(z_{3}\right)<0, h^{\prime}\left(z_{4}\right)>0$. It follows from theorem 7 that

$$
\frac{\mathrm{d} \zeta\left(\tau_{1}^{(j)}\right)}{\mathrm{d} \tau}<0, \frac{\mathrm{d} \zeta\left(\tau_{2}^{(j)}\right)}{\mathrm{d} \tau}>0, \quad \frac{\mathrm{d} \zeta\left(\tau_{3}^{(j)}\right)}{\mathrm{d} \tau}<0, \frac{\mathrm{d} \zeta\left(\tau_{4}^{(j)}\right)}{\mathrm{d} \tau}>0, \quad j=0,1,2, \ldots
$$

which implies that stability switches may appear as $\tau$ increasing.

\section{Stability and Direction of the Hopf Bifurcation}

In this section, we shall study the direction of the Hopf bifurcations and stability of bifurcating periodic solutions by applying the normal theory and the center manifold theorem introduced by Hassard et al. [7].

Let $u_{1}=j-j^{*}, u_{2}=s-s^{*}, u_{3}=i-i^{*}, u_{4}=r-r^{*}, \widetilde{u}_{i}(t)=u_{i}(\tau t)$, $\tau=\nu+\tau^{*}$ and dropping the bars for simplification of notations, system (2.3) 
becomes an functional differential equation in $C=C\left([-1,0], \mathbb{R}^{4}\right)$ as

$$
\left\{\begin{array}{l}
\frac{\mathrm{d} u_{1}(t)}{\mathrm{d} t}=\left(\tau^{*}+\nu\right)\left[-u_{1}(t)+u_{2}(t)+u_{3}(t)+u_{4}(t)\right], \\
\frac{\mathrm{d} u_{2}(t)}{\mathrm{d} t}=\left(\tau^{*}+\nu\right)\left\{a u_{1}(t)-b_{2} u_{2}(t)-\frac{\beta_{1}\left[u_{2}(t)+s^{*}\right]\left[u_{3}(t-1)+i^{*}\right]}{1+\alpha_{1} i^{*}+\alpha_{1} u_{3}(t-1)}\right. \\
\left.+\delta_{1} u_{4}(t)+\frac{\beta_{1} s^{*} i^{*}}{1+\alpha_{1} i^{*}}\right\} \\
\frac{\mathrm{d} u_{3}(t)}{\mathrm{d} t}=\left(\tau^{*}+\nu\right)\left\{\frac{\beta_{1}\left[u_{2}(t)+s^{*}\right]\left[u_{3}(t-1)+i^{*}\right]}{1+\alpha_{1} i^{*}+\alpha_{1} u_{3}(t-1)}-b_{2} u_{3}(t)-b_{2} i^{*}\right\}, \\
\frac{\mathrm{d} u_{4}(t)}{\mathrm{d} t}=\left(\tau^{*}+\nu\right)\left[\gamma_{1} u_{3}(t)-b_{3} u_{4}(t)\right] .
\end{array}\right.
$$

Expanding the nonlinear part by Taylor expansion, we obtain

$$
\left\{\begin{aligned}
\frac{\mathrm{d} u_{1}(t)}{\mathrm{d} t}= & \left(\tau^{*}+\nu\right)\left[-u_{1}(t)+u_{2}(t)+u_{3}(t)+u_{4}(t)\right], \\
\frac{\mathrm{d} u_{2}(t)}{\mathrm{d} t}= & \left(\tau^{*}+\nu\right)\left[a u_{1}(t)-\left(b_{2}+\frac{\beta_{1} i^{*}}{1+\alpha_{1} i^{*}}\right) u_{2}(t)-\frac{\beta_{1} s^{*}}{\left(1+\alpha_{1} i^{*}\right)^{2}} u_{3}(t-1)\right. \\
& +\delta_{1} u_{4}(t) \\
& -\frac{\beta_{1}}{\left(1+\alpha_{1} i^{*}\right)^{2}} u_{2}(t) u_{3}(t-1)+\frac{\alpha_{1} \beta_{1} s^{*}}{\left(1+\alpha_{1} i^{*}\right)^{3}} u_{3}^{2}(t-1) \\
& +\frac{\alpha_{1} \beta_{1}}{\left(1+\alpha_{1} i^{*}\right)^{3}} u_{2}(t) u_{3}^{2}(t-1) \\
& -\frac{\alpha_{1}^{2} \beta_{1} s^{*}}{\left(1+\alpha_{1} i^{*}\right)^{4}} u_{3}^{3}(t-1)-\frac{\alpha_{1}^{2} \beta_{1}}{\left(1+\alpha_{1} i^{*}\right)^{4}} u_{2}(t) u_{3}^{3}(t-1) \\
& +\ldots+(-1)^{n} \frac{\alpha_{1}^{n+1} \beta_{1} s^{*}}{\left(1+\alpha_{1} i^{*}\right)^{n+3}} u_{3}^{n+2}(t-1) \\
& \left.+(-1)^{n} \frac{\alpha_{1}^{n+1} \beta_{1}}{\left(1+\alpha_{1} i^{*}\right)^{n+3}} u_{2}(t) u_{3}^{n+2}(t-1)+\ldots\right], \\
\frac{\mathrm{d} u_{3}(t)}{\mathrm{d} t}= & \left(\tau^{*}+\nu\right)\left[\frac{\beta_{1} i^{*}}{1+\alpha_{1} i^{*}} u_{2}(t)+\frac{\beta_{1} s^{*}}{\left(1+\alpha_{1} i^{*}\right)^{2}} u_{3}(t-1)\right. \\
& -b_{2} u_{3}(t)+\frac{\beta_{1}}{\left(1+\alpha_{1} i^{*}\right)^{2}} u_{2}(t) u_{3}(t-1) \\
& -\frac{\alpha_{1} \beta_{1} s^{*}}{\left(1+\alpha_{1} i^{*}\right)^{3}} u_{3}^{2}(t-1)-\frac{\alpha_{1} \beta_{1}}{\left(1+\alpha_{1} i^{*}\right)^{3}} u_{2}(t) u_{3}^{2}(t-1) \\
& +\frac{\alpha_{1}^{2} \beta_{1} s^{*}}{\left(1+\alpha_{1} i^{*}\right)^{4}} u_{3}^{3}(t-1) \\
& +\frac{\alpha_{1}^{2} \beta_{1}}{\left(1+\alpha_{1} i^{*}\right)^{4}} u_{2}(t) u_{3}^{3}(t-1)+\ldots+(-1)^{n+1} \frac{\alpha_{1}^{n+1} \beta_{1} s^{*}}{\left(1+\alpha_{1} i^{*}\right)^{n+3}} u_{3}^{n+2}(t-1) \\
& \left.+(-1)^{n+1} \frac{\alpha_{1}^{n+1} \beta_{1}}{\left(1+\alpha_{1} i^{*}\right)^{n+3}} u_{2}(t) u_{3}^{n+2}(t-1)+\ldots\right], \\
\frac{\mathrm{d} u_{4}(t)}{\mathrm{d} t}= & \left(\tau^{*}+\nu\right)\left[\gamma_{1} u_{3}(t)-b_{3} u_{4}(t)\right] .
\end{aligned}\right.
$$

where $n=-1,0,1 \cdots$. We denote the above system by

$$
\dot{u}(t)=L_{\nu}\left(u_{t}\right)+f\left(\nu, u_{t}\right),
$$

where $u(t)=\left(u_{1}(t), u_{2}(t), u_{3}(t), u_{4}(t)\right)^{\mathrm{T}} \in \mathbb{R}^{4}$, and $L_{\nu}: C \rightarrow \mathbb{R}^{4}, f: \mathbb{R} \times C \rightarrow$ 
$\mathbb{R}^{4}$ are given, respectively, by

$$
\begin{aligned}
L_{\nu}(\phi)= & \left(\tau^{*}+\nu\right)\left(\begin{array}{cccc}
-1 & 1 & 1 & 1 \\
a & -\left(b_{2}+\frac{\beta_{1} i^{*}}{1+\alpha_{1} i^{*}}\right) & 0 & \delta_{1} \\
0 & \frac{\beta_{1} i^{*}}{1+\alpha_{1} i^{*}} & -b_{2} & 0 \\
0 & 0 & \gamma_{1} & -b_{3}
\end{array}\right)\left(\begin{array}{l}
\phi_{1}(0) \\
\phi_{2}(0) \\
\phi_{3}(0) \\
\phi_{4}(0)
\end{array}\right) \\
& +\left(\tau^{*}+\nu\right)\left(\begin{array}{cccc}
0 & 0 & 0 & 0 \\
0 & 0 & -\frac{\beta_{1} s^{*}}{\left(1+\alpha_{1} *^{*}\right)^{2}} & 0 \\
0 & 0 & \frac{\beta_{1} s^{*}}{\left(1+\alpha_{1} i^{*}\right)^{2}} & 0 \\
0 & 0 & 0 & 0
\end{array}\right)\left(\begin{array}{c}
\phi_{1}(-1) \\
\phi_{2}(-1) \\
\phi_{3}(-1) \\
\phi_{4}(-1)
\end{array}\right)
\end{aligned}
$$

and

$$
\begin{aligned}
f(\nu, \phi)= & \left(\tau^{*}+\nu\right)\left\{\frac{\beta_{1} \phi_{2}(0)}{\left(1+\alpha_{1} i^{*}\right)^{2}}\left(\begin{array}{c}
0 \\
\phi_{3}(-1) \\
-\phi_{3}(-1) \\
0
\end{array}\right)+\frac{\alpha_{1} \beta_{1} s^{*}}{\left(1+\alpha_{1} i^{*}\right)^{3}}\left(\begin{array}{c}
0 \\
\phi_{3}^{2}(-1) \\
-\phi_{3}^{2}(-1) \\
0
\end{array}\right)\right. \\
& +\frac{\alpha_{1} \beta_{1} \phi_{2}(0)}{\left(1+\alpha_{1} i^{*}\right)^{3}}\left(\begin{array}{c}
0 \\
\phi_{3}^{2}(-1) \\
-\phi_{3}^{2}(-1) \\
0
\end{array}\right)+\ldots+(-1)^{n} \frac{\alpha_{1}^{n+1} \beta_{1} s^{*}}{\left(1+\alpha_{1} i^{*}\right)^{n+3}}\left(\begin{array}{c}
\phi_{3}^{n+2}(-1) \\
-\phi_{3}^{n+2}(-1) \\
0
\end{array}\right) \\
& \left.+(-1)^{n} \frac{\alpha_{1}^{n+1} \beta_{1} \phi_{2}(0)}{\left(1+\alpha_{1} i^{*}\right)^{n+3}}\left(\begin{array}{c}
\phi_{3}^{n+2}(-1) \\
-\phi_{3}^{n+2}(-1) \\
0
\end{array}\right)+\ldots\right\} .
\end{aligned}
$$

By the Riesz representation theorem, there exists a function $\eta(\theta, \nu)$ of bounded variation for $\theta \in[-1,0]$, such that

$$
L_{\nu}(\phi)=\int_{-1}^{0} \mathrm{~d} \eta(\theta, \nu) \phi(\theta), \quad \text { for } \phi \in C .
$$

In fact, we can choose

$$
\begin{aligned}
& \eta(\theta, \nu)=\left(\tau^{*}+\nu\right)\left(\begin{array}{cccc}
-1 & 1 & 1 & 1 \\
a & -\left(b_{2}+\frac{\beta_{1} i^{*}}{1+\alpha i_{1}}\right) & 0 & \delta_{1} \\
0 & \frac{\beta_{1} i^{*}}{1+\alpha_{1} i^{*}} & -b_{2} & 0 \\
0 & 0 & \gamma_{1} & -b_{3}
\end{array}\right) \delta(\theta) \\
& -\left(\tau^{*}+\nu\right)\left(\begin{array}{cccc}
0 & 0 & 0 & 0 \\
0 & 0 & -\frac{\beta_{1} s^{*}}{\left(1+\alpha_{i} i^{*}\right)^{2}} & 0 \\
0 & 0 & \frac{\beta_{1} s^{*}}{\left(1+\alpha_{1} i^{*}\right)^{2}} & 0 \\
0 & 0 & 0 & 0
\end{array}\right) \delta(\theta+1) .
\end{aligned}
$$


where $\delta$ denote the Dirac delta function. For $\phi \in C\left([-1,0], \mathbb{R}^{4}\right)$, define

and

$$
A(\nu) \phi= \begin{cases}\frac{\mathrm{d} \phi(\theta)}{\mathrm{d} \theta}, & \theta \in[-1,0), \\ \int_{-1}^{0} \mathrm{~d} \eta(\theta, \nu) \phi(\theta), & \theta=0 .\end{cases}
$$

$$
R(\nu)(\phi)= \begin{cases}0, & \theta \in[-1,0), \\ f(\nu, \phi), & \theta=0 .\end{cases}
$$

Then, system (4.1) is equivalent to

$$
\dot{u}(t)=A(\nu) u_{t}+R(\nu) u_{t},
$$

where $u_{t}=u(t+\theta)$ for $\theta \in[-1,0]$.

For $\psi \in C\left([0,1],\left(\mathbb{R}^{4}\right)^{*}\right)$, define

$$
A^{*} \psi(s)= \begin{cases}-\frac{\mathrm{d} \psi(s)}{\mathrm{d} s}, & s \in(0,1], \\ \int_{-1}^{0} \psi(-t) \mathrm{d} \eta(t, 0), & s=0 .\end{cases}
$$

and a bilinear inner product

$$
\langle\psi(s), \phi(\theta)\rangle=\bar{\psi}(0) \phi(0)-\int_{-1}^{0} \int_{\sigma=0}^{\theta} \bar{\psi}(\sigma-\theta) \mathrm{d} \eta(\theta) \phi(\sigma) \mathrm{d} \sigma .
$$

where $\eta(\theta)=\eta(\theta, 0)$. Then $A(0)$ and $A^{*}$ are adjoint operators. By the discussion in Section 3.3, we know that $\pm i \xi^{*} \tau^{*}$ are eigenvalues of $A(0)$. Hence, they are also eigenvalues of $A^{*}$. We first need to compute the eigenvectors of $A(0)$ and $A^{*}$ corresponding to $i \xi^{*} \tau^{*}$ and $-i \xi^{*} \tau^{*}$, respectively.

Assume that $q(\theta)=\left(1, q_{1}, q_{2}, q_{3}\right)^{\mathrm{T}} e^{i \xi^{*} \tau^{*} \theta}$ is the eigenvector of $A(0)$ corresponding to $i \xi^{*} \tau^{*}$, then $A(0) q(\theta)=i \xi^{*} \tau^{*} q(\theta)$. Then from the definition of $A(0)$ and (4.2), (4.4), (4.5), and for $q(-1)=q(0) e^{-i \xi^{*} \tau^{*}}$, we have

$$
\begin{array}{r}
\tau^{*}\left(\begin{array}{cccc}
-1 & 1 & 1 & 1 \\
a & -\left(b_{2}+\frac{\beta_{1} i^{*}}{1+\alpha_{1} i^{*}}\right) & -\frac{\beta_{1} s^{*} e^{-i \xi^{*} \tau^{*}}}{\left(1+\alpha_{1} i^{*}\right)^{2}} & \delta_{1} \\
0 & \frac{\beta_{1} i^{*}}{1+\alpha_{1} i^{*}} & -b_{2}+\frac{\beta_{1} s^{*} e^{-i \xi^{*} \tau^{*}}}{\left(1+\alpha_{1} i^{*}\right)^{2}} & 0 \\
0 & 0 & \gamma_{1} & -b_{3}
\end{array}\right) \\
\quad=i \xi^{*} \tau^{*}\left(\begin{array}{c}
1 \\
q_{1}(0) \\
q_{2}(0) \\
q_{3}(0)
\end{array}\right) \\
\\
\left.\quad \begin{array}{c}
1 \\
q_{1}(0) \\
q_{2}(0) \\
q_{3}(0)
\end{array}\right)
\end{array}
$$


Then we obtain

$$
\left\{\begin{array}{l}
q_{1}=\frac{\left(i \xi^{*}+1\right)\left(i \xi^{*}+b_{3}\right)\left[\left(i \xi^{*}+b_{2}\right)\left(1+\alpha_{1} i^{*}\right)-b_{2} e^{-i \xi^{*} \tau^{*}}\right]}{\left[\left(i \xi^{*}+b_{2}\right)\left(i \xi^{*}+b_{3}\right)\left(1+\alpha_{1} i^{*}\right)+\beta_{1} i^{*}\left(i \xi^{*}+b_{3}\right)+\beta_{1} \gamma_{1} i^{*}-b_{2}\left(b_{3}+i \xi^{*}\right) e^{\left.-i \xi^{*} \tau^{*}\right]}\right.} \\
q_{2}=\frac{\left(i \xi^{*}+1\right)\left(i \xi^{*}+b_{3}\right) \beta_{1} i^{*}}{\left[\left(i \xi^{*}+b_{2}\right)\left(i \xi^{*}+b_{3}\right)\left(1+\alpha_{1} i^{*}\right)+\beta_{1} i^{*}\left(i \xi^{*}+b_{3}\right)+\beta_{1} \gamma_{1} i^{*}-b_{2}\left(b_{3}+i \xi^{*}\right) e^{\left.-i \xi^{*} \tau^{*}\right]}\right.} \\
q_{3}=\frac{\beta_{1} \gamma_{1} i^{*}\left(i \xi^{*}+1\right)}{\left[\left(i \xi^{*}+b_{2}\right)\left(i \xi^{*}+b_{3}\right)\left(1+\alpha_{1} i^{*}\right)+\beta_{1} i^{*}\left(i \xi^{*}+b_{3}\right)+\beta_{1} \gamma_{1} i^{*}-b_{2}\left(b_{3}+i \xi^{*}\right) e^{\left.-i \xi^{*} \tau^{*}\right]}\right.}
\end{array}\right.
$$

Similarly, we can calculate the eigenvector $q^{*}(s)=D\left(1, q_{1}^{*}, q_{2}^{*}, q_{3}^{*}\right)^{\mathrm{T}} e^{i \xi^{*} \tau^{*} s}$ of $A^{*}$ corresponding to $-i \xi^{*} \tau^{*}$. Where

$$
\left\{\begin{array}{l}
q_{1}^{*}=\frac{-i \xi^{*}+1}{a} \\
q_{2}^{*}=\frac{-i \xi^{*} \delta_{1}+a+\delta_{1}}{a\left(-i \xi^{*}+b_{3}\right)} \\
q_{3}^{*}=\frac{\left[\left(-i \xi^{*}+b_{2}\right)\left(1+\alpha_{1} i^{*}\right)+\beta_{1} i^{*}\right]\left(-i \xi^{*}+1\right)-a\left(1+\alpha_{1} i^{*}\right)}{a \beta_{1} i^{*}}
\end{array}\right.
$$

We normalize $q$ and $q^{*}$ by the condition $\left\langle q^{*}(s), q(\theta)\right\rangle=1$. Clearly,

$$
\left\langle q^{*}(s), \bar{q}(\theta)\right\rangle=0 .
$$

In order to assure $\left\langle q^{*}(s), q(\theta)\right\rangle=1$, we need to determine the value of $D$. By (4.7), we have

$$
\begin{aligned}
& \langle\psi(s), \phi(\theta)\rangle=\bar{D}\left(1, \bar{q}_{1}^{*}, \bar{q}_{2}^{*}, \bar{q}_{3}^{*}\right)\left(1, q_{1}, q_{2}, q_{3}\right)^{\mathrm{T}} \\
& -\int_{-1}^{0} \int_{\sigma=0}^{\theta} \bar{D}\left(1, \bar{q}_{1}^{*}, \bar{q}_{2}^{*}, \bar{q}_{3}^{*}\right) e^{-i \xi^{*} \tau^{*}(\sigma-\theta)} \mathrm{d} \eta(\theta)\left(1, q_{1}, q_{2}, q_{3}\right)^{\mathrm{T}} e^{i \xi^{*} \tau^{*} \sigma} \mathrm{d} \sigma \\
= & \bar{D}\left\{1+q_{1} \bar{q}_{1}^{*}+q_{2} \bar{q}_{2}^{*}+q_{3} \bar{q}_{3}^{*}-\int_{-1}^{0}\left(1, \bar{q}_{1}^{*}, \bar{q}_{2}^{*}, \bar{q}_{3}^{*}\right) \theta e^{i \xi^{*} \tau^{*} \theta} \mathrm{d} \eta(\theta)\left(1, q_{1}, q_{2}, q_{3}\right)^{\mathrm{T}}\right\} \\
= & \bar{D}\left\{1+q_{1} \bar{q}_{1}^{*}+q_{2} \bar{q}_{2}^{*}+q_{3} \bar{q}_{3}^{*}+\tau^{*} q_{2} e^{-i \xi^{*} \tau^{*}}\left(-\bar{q}_{1}^{*}+\bar{q}_{2}^{*}\right) \frac{\beta_{1} s^{*}}{\left(1+\alpha_{1} i^{*}\right)^{2}}\right\} .
\end{aligned}
$$

Therefore, we can choose $D$ as

$$
D=\frac{1}{1+\bar{q}_{1} q_{1}^{*}+\bar{q}_{2} q_{2}^{*}+\bar{q}_{3} q_{3}^{*}+\tau^{*} \bar{q}_{2} e^{i \xi^{*} \tau^{*}}\left(-q_{1}^{*}+q_{2}^{*}\right) \frac{\beta_{1} s^{*}}{\left(1+\alpha_{1} i^{*}\right)^{2}}} .
$$

Following the algorithms given in [7] and using similar computation process in [11], we can get that the coefficients which will be used to determine the important quantities

$$
\begin{aligned}
g_{20}= & \frac{2 \beta_{1} \tau^{*} \bar{D}}{\left(1+\alpha_{1} i^{*}\right)^{2}}\left(-\bar{q}_{1}^{*}+\bar{q}_{2}^{*}\right) q_{1} q_{2} e^{-i \xi^{*} \tau^{*}}+\frac{2 \alpha_{1} \beta_{1} s^{*} \tau^{*} \bar{D}}{\left(1+\alpha_{1} i^{*}\right)^{3}}\left(\bar{q}_{1}^{*}-\bar{q}_{2}^{*}\right) q_{2}^{2} e^{-2 i \xi^{*} \tau^{*}} \\
g_{11}= & \frac{2 \beta_{1} \tau^{*} \bar{D}}{\left(1+\alpha_{1} i^{*}\right)^{2}}\left(-\bar{q}_{1}^{*}+\bar{q}_{2}^{*}\right) \Re\left\{q_{1} \bar{q}_{2} e^{i \xi^{*} \tau^{*}}\right\}+\frac{2 \alpha_{1} \beta_{1} s^{*} \tau^{*} \bar{D}}{\left(1+\alpha_{1} i^{*}\right)^{3}}\left(\bar{q}_{1}^{*}-\bar{q}_{2}^{*}\right)\left|q_{2}\right|^{2} \\
g_{02}= & \frac{2 \beta_{1} \tau^{*} \bar{D}}{\left(1+\alpha_{1} i^{*}\right)^{2}}\left(-\bar{q}_{1}^{*}+\bar{q}_{2}^{*}\right) \bar{q}_{1} \bar{q}_{2} e^{i \xi^{*} \tau^{*}}+\frac{2 \alpha_{1} \beta_{1} s^{*} \tau^{*} \bar{D}}{\left(1+\alpha_{1} i^{*}\right)^{3}}\left(\bar{q}_{1}^{*}-\bar{q}_{2}^{*}\right) \bar{q}_{2}^{2} e^{2 i \xi^{*} \tau^{*}} ; \\
g_{21}= & \frac{\beta_{1} \tau^{*} \bar{D}}{\left(1+\alpha_{1} i^{*}\right)^{2}}\left(-\bar{q}_{1}^{*}+\bar{q}_{2}^{*}\right)\left[\bar{q}_{2} e^{i \xi^{*} \tau^{*}} W_{20}^{(2)}(0)+2 q_{2} e^{-i \xi^{*} \tau^{*}} W_{11}^{(2)}(0)+\bar{q}_{1} W_{20}^{(3)}(-1)\right. \\
& \left.+2 q_{1} W_{11}^{(3)}(-1)\right]+\frac{2 \alpha_{1} \beta_{1} s^{*} \tau^{*} \bar{D}}{\left(1+\alpha_{1} i^{*}\right)^{3}}\left(\bar{q}_{1}^{*}-\bar{q}_{2}^{*}\right)\left[\bar{q}_{2} e^{i \xi^{*} \tau^{*}} W_{20}^{(3)}(-1)\right. \\
& \left.+2 q_{2} e^{-i \xi^{*} \tau^{*}} W_{11}^{(3)}(-1)\right]+\frac{2 \alpha_{1} \beta_{1} s^{*} \tau^{*} \bar{D}}{\left(1+\alpha_{1} i^{*}\right)^{3}}\left(\bar{q}_{1}^{*}-\bar{q}_{2}^{*}\right)\left[\bar{q}_{1} q_{2}^{2} e^{-2 i \xi^{*} \tau^{*}}+2 q_{1}\left|q_{2}\right|^{2}\right] .
\end{aligned}
$$


where

$$
\begin{aligned}
W_{20}(\theta) & =\frac{i g_{20}}{\xi^{*} \tau^{*}} q(0) e^{i \xi^{*} \tau^{*} \theta}+\frac{i \bar{g}_{02}}{3 \xi^{*} \tau^{*}} \bar{q}(0) e^{-i \xi^{*} \tau^{*} \theta}+\left[W_{20}(0)+\frac{g_{20}}{i \xi^{*} \tau^{*}} q(0)\right. \\
& \left.+\frac{\bar{g}_{02}}{3 i \xi^{*} \tau^{*}} \bar{q}(0)\right] e^{2 i \xi^{*} \tau^{*} \theta} \triangleq \frac{i g_{20}}{\xi^{*} \tau^{*}} q(0) e^{i \xi^{*} \tau^{*} \theta}+\frac{i \bar{g}_{02}}{3 \xi^{*} \tau^{*}} \bar{q}(0) e^{-i \xi^{*} \tau^{*} \theta} \\
& +E_{1} e^{2 i \xi^{*} \tau^{*} \theta}
\end{aligned}
$$

and

$$
W_{11}(\theta)=\frac{i g_{11}}{\xi^{*} \tau^{*}} \bar{q}(0) e^{-i \xi^{*} \tau^{*} \theta}+E_{2},
$$

Moreover $E_{1}, E_{2}$ satisfy the following equations, respectively,

$$
\begin{aligned}
& \left(\begin{array}{cccc}
2 i \xi^{*}+1 & -1 & -1 & -1 \\
-a & 2 i \xi^{*}+b_{1}+\frac{\beta_{1} i^{*}}{1+\alpha_{1} i^{*}} & \frac{\beta_{1} s^{*} e^{-2 i \xi^{*} \tau^{*}}}{\left(1+\alpha_{1} i^{*}\right)^{2}} & -\delta_{1} \\
0 & -\frac{\beta_{1} i^{*}}{1+\alpha_{1} i^{*}} & 2 i \xi^{*}+b_{2}-\frac{\beta_{1} s^{*} e^{-2 i \xi^{*} \tau^{*}}}{\left(1+\alpha_{1} i^{*}\right)^{2}} & 0 \\
0 & 0 & -\gamma_{1} & 2 i \xi^{*}+b_{3}
\end{array}\right) E_{1} \\
& =\frac{2 \beta_{1} q_{1} q_{2} e^{-i \xi^{*} \tau^{*}}}{\left(1+\alpha_{1} i^{*}\right)^{2}}(0,-1,1,0)^{\mathrm{T}}+\frac{2 \alpha_{1} \beta_{1} s^{*} q_{2}^{2} e^{-2 i \xi^{*} \tau^{*}}}{\left(1+\alpha_{1} i^{*}\right)^{3}}(0,1,-1,0)^{\mathrm{T}}
\end{aligned}
$$

and

$$
\begin{aligned}
& \left(\begin{array}{cccc}
1 & -1 & -1 & -1 \\
-a & b_{1}+\frac{\beta_{1} i^{*}}{1+\alpha_{1} i^{*}} & \frac{\beta_{1} s^{*}}{\left(1+\alpha_{1} i^{*}\right)^{2}} & -\delta_{1} \\
0 & -\frac{\beta_{1} i^{*}}{1+\alpha_{1} i^{*}} & b_{2}-\frac{\beta_{1} s^{*}}{\left(1+\alpha_{1} i^{*}\right)^{2}} & 0 \\
0 & 0 & -\gamma_{1} & b_{3}
\end{array}\right) E_{2} \\
& =\frac{2 \beta_{1} \tau^{*} \Re\left\{q_{1} \bar{q}_{2} e^{i \xi^{*} \tau^{*}}\right\}}{\left(1+\alpha_{1} i^{*}\right)^{2}}(0,-1,1,0)^{\mathrm{T}}+\frac{2 \alpha_{1} \beta_{1} s^{*} \tau^{*}\left|q_{2}\right|^{2}}{\left(1+\alpha_{1} i^{*}\right)^{3}}(0,1,-1,0)^{\mathrm{T}}
\end{aligned}
$$

Because each $g_{i j}$ is expressed by the parameters and delay in (4.8), we can compute the following quantities:

$$
\begin{array}{ll}
c_{1}(0)=\frac{i}{2 \xi^{*} \tau^{*}}\left(g_{20} g_{11}-2\left|g_{11}\right|^{2}-\frac{\left|g_{02}\right|}{3}\right)+\frac{g_{21}}{2}, & \nu_{2}=-\frac{\operatorname{Re}\left\{c_{1}(0)\right\}}{\operatorname{Re}\left\{\frac{\mathrm{d} \lambda\left(\tau^{*}\right)}{\mathrm{d} \tau}\right\}}, \\
\beta_{2}=2 \operatorname{Re}\left\{c_{1}(0)\right\}, & T_{2}=-\frac{\operatorname{Re}\left\{c_{1}(0)\right\}+\nu_{2} \operatorname{Re}\left\{\frac{\mathrm{d} \lambda\left(\tau^{*}\right)}{\mathrm{d} \tau}\right\}}{\xi^{*} \tau^{*}} .
\end{array}
$$

By the result of Hassard et al.[7], we have the following:

Theorem 5.1 In (5.11), the following results hold:

(i) the sign of $\nu_{2}$ determines the directions of the Hopf bifurcation: if $\nu_{2}>0\left(\nu_{2}<0\right)$, then the Hopf bifurcation is supercritical(subcritical) and the bifurcating periodic solutions exist for $\tau>\tau^{*}\left(\tau<\tau^{*}\right)$;

(ii)the sign of beta $a_{2}$ determines the stability of the bifurcating periodic solutions: the bifurcating periodic solutions are stable(unstable) if $\beta_{2}<0\left(\beta_{2}>0\right.$ );

(iii)the sign of $T_{2}$ determines the period of the bifurcating periodic solutions: the period increasing (decreasing) if $\beta_{2}>0\left(\beta_{2}<0\right)$. 


\section{Numerical Simulations}

In this paper, we have investigated the dynamic behavior of a delayed stagestructured SIRS epidemic model and a nonlinear incidence rates. Real epidemic data show regular periodic fluctuations in disease incidence, but most simple deterministic models for infectious diseases predict convergence to a unique stable endemic equilibrium, so it is important to examine under what circumstances periodic fluctuations in disease incidence can arise. In our paper, the stability of disease-free equilibrium was verified by Routh-Hurwitz criterion and LaSalle's invariance principle. We researched the existence of Hopf bifurcation and obtained the stability and direction of the Hopf bifurcation by using the normal theory and the center manifold theorem.

As an example, we present some numerical results of system (2.1) at different values $\tau$. We consider the following set of the parameters: $a=0.4 ; b_{1}=$ $0.6 ; b_{2}=2.5 ; b_{3}=0.6 ; \alpha_{1}=0.001 ; \beta_{1}=1 ; \delta_{1}=0.1 ; \gamma_{1}=0.5 ; \tau=10$ and $J(0)=0.4 ; S(0)=0.2 ; I(0)=0.2 ; R(0)=0.2$. By directly computing, we obtain $\mathscr{R}_{0}=0.8<1$. According to theorem 3 , the disease-free equilibrium $E_{0}$ is locally asymptotically stable for all $\tau \geq 0$ (Fig.1 and Fig.2).
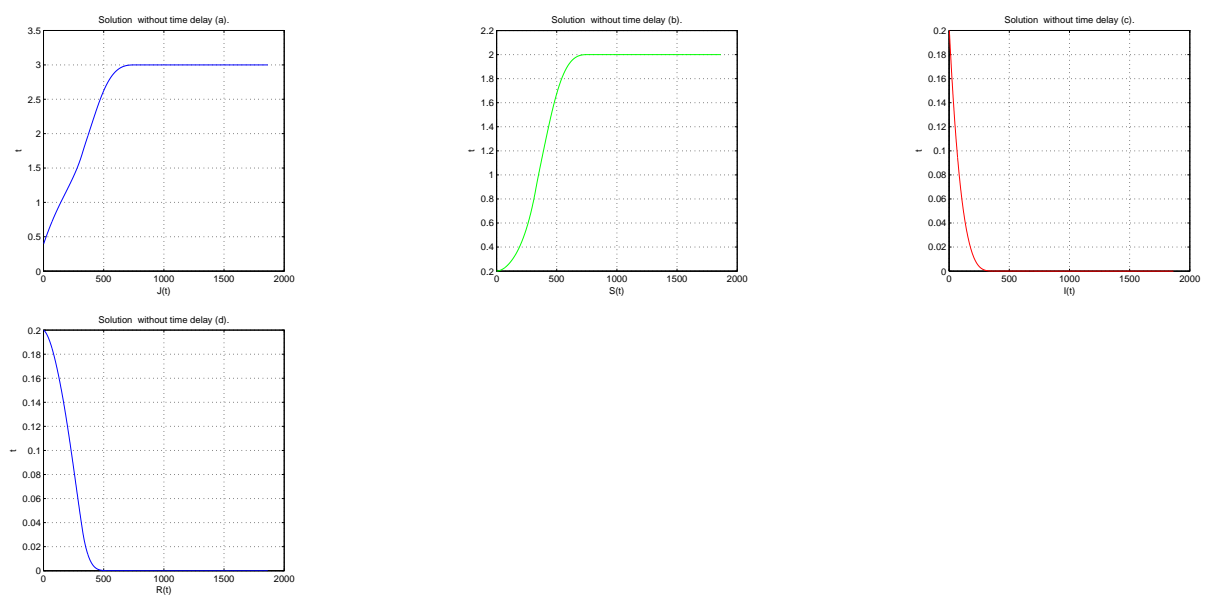

Figure 1: Figs (a)-(d) and (i) are system (2.1) with initial condition $J(0)=0.4 ; S(0)=0.2 ; I(0)=0.2 ; R(0)=0.2$ and without time delay, the disease-free equilibrium $E_{0}$ is locally asymptotically stable. 

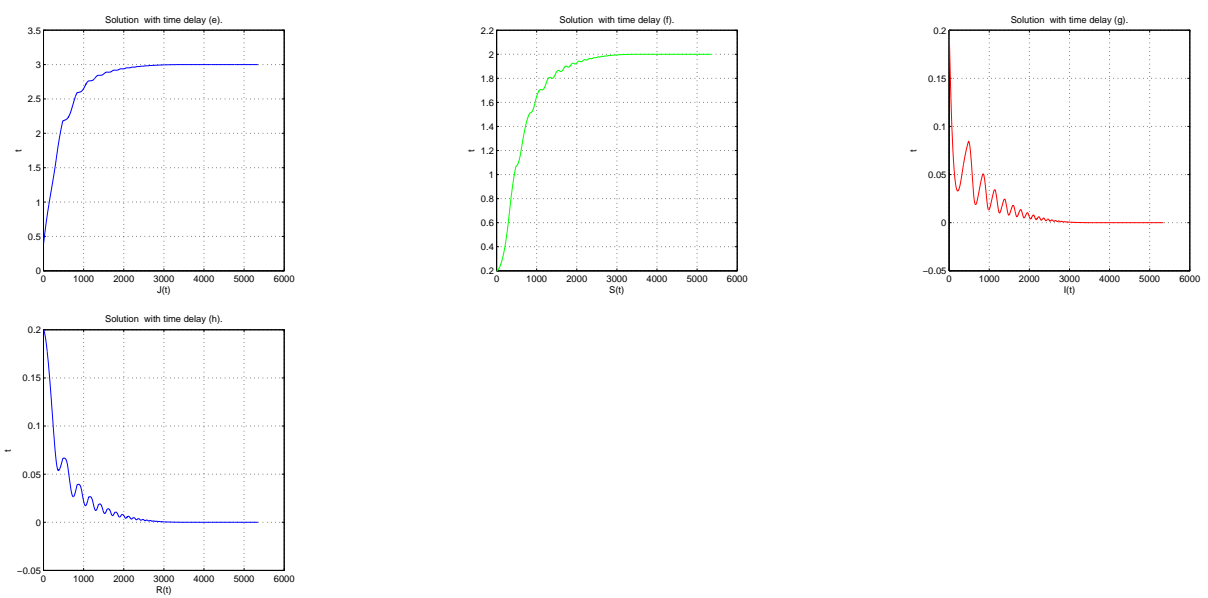

Figure 2: Figs (e)-(h) and (j) are system (2.1) with initial condition $J(0)=0.4 ; S(0)=0.2 ; I(0)=0.2 ; R(0)=0.2$ and with time delay $\tau=10$, the disease-free equilibrium $E_{0}$ is also locally asymptotically stable.

\section{Acknowledgments}

This work is supported by Natural Science Foundation of Shanxi province (2013011002-2).

\section{References}

[1] Z.G. Bai, A periodic age-structured epidemic model with a wide class of incidence rates, Journal of Mathematical Analysis and Applications, 393( 2012), 367-376.

[2] K Cooke, P. van den Driessche, X Zou, Interaction of maturation delay and nonlinear birth in population and epidemic models, J. Math. Biol, 39(1999), 332-352.

[3] G.H. Cui, X.P. Yan, Stability and bifurcation analysis on a three-species food chain system with two delays, Commun Nonlinear Sci Numer Simulat, 16(2011), 3704-3720.

[4] X.H. Ding, W.X. Li, Local Hopf bifurcation and global existence of periodic solutions in a kind of physiological system, Nonlinear Analysis: RealWorld Applications, 8(2007), 1459-1471. 
[5] O. Diekmann, J.A.P. Heesterbeek, J.A.J. Metz, On the definition and the computation of the basic reproduction ratio $\mathscr{R}_{0}$ in models for infectious diseases in heterogeneous populations, J. Math. Biol, 28(1990), 365-382.

[6] P. van den Driessche, J.Watmough, Reproduction numbers and subthreshold endemic equilibria for compartmental models of disease transmission, Math. Biosci, 180(2002), 29C48.

[7] B. Hassard, N. Kazarinoff, Y. Wan, Theory and Applications of Hopf Bifurcation, Cambridge University Press, Cambridge, 1981.

[8] W.O. Kermack, A.G. Mckendrick, Contribution to the mathematical theory of epidemics, Proc. R. Soc. Lond. Ser. A, 115(1927), 700-721.

[9] Y Kuang, Delay Differential Equations with Application in Population Dynamics, Academic press, INC, San Diego, CA, 1993.

[10] F Li, H.W. Li, Hopf bifurcation of a predator-prey model with time delay and stage structure for the prey, Mathematical and Computer Modelling, $55(2012), 672-679$.

[11] J.J. Wei, S.G. Ruan, Stability and bifurcation in a neural network model with two delays, Physica D, 130(1999), 255-272.

[12] $\mathrm{R} \mathrm{Xu}$, Global dynamics of a predator-prey model with time delay and stage structure for the prey, Nonlinear Analysis: Real World Applications, 12(2011), 2151-2162.

[13] H.Y. Yang, Y.P. Tian, Hopf bifurcation in REM algorithm with communication delay, Chaos, Solitons and Fractals, 25(2005), 1093-1105.

[14] T.L. Zhang, J.L. Liu, Z.D. Teng, Stability of Hopf bifurcation of a delayed SIRS epidemic model with stage structure, Nonlinear Analysis: Real World Applications, 11(2010), 293-306. 
